\title{
Treatment Patterns and Costs in Biologic DMARD-Naive Patients with Rheumatoid Arthritis Initiating Etanercept or Adalimumab with or Without Methotrexate
}

\author{
Joseph Tkacz, MS; Mahdi Gharaibeh, PharmD, MS, PhD; Kathryn Henderson DeYoung, MS;
} Kathleen Wilson, MPH; David Collier, MD; and Hafiz Oko-osi, MS, RPh

\begin{abstract}
BACKGROUND: Etanercept (ETN) and adalimumab (ADA) are tumor necrosis factor inhibitors indicated for treatment of moderate to severe rheumatoid arthritis (RA) and are used as monotherapy or in combination with conventional disease-modifying antirheumatic drugs (DMARDs) such as methotrexate (MTX). Data on treatment patterns and costs of ETN and ADA as monotherapies or in combination therapy with MTX are lacking in biologic DMARD (bDMARD)-naive patients with RA.
\end{abstract}

OBJECTIVE: To evaluate treatment patterns and costs of ETN and ADA monotherapy and combination therapy in bDMARD-naive patients with RA.

METHODS: Data from adult bDMARD-naive patients with RA were evaluated according to index therapy (ADA or ETN as monotherapy or combination therapy with MTX) in a retrospective cohort study using the IBM MarketScan Commercial Claims and Encounters and Medicare Supplemental Databases from January 1, 2010, to June 30, 2017.

Participants were bDMARD-naive for $\geq 12$ months before initial ETN or ADA pharmacy claim (index date) and had continuous enrollment for $\geq 12$ months pre-index and 24 months post-index. Combination therapy cohorts had an MTX claim within 30 days of the index date. Outcomes included persistence (no treatment changes or gap [ $\geq 60$ days]); modifications to index therapy (discontinuation or switching without prior gap, restarting as switch or restart after gap, or MTX initiation/discontinuation); and mean total bDMARD costs for 2 years post-index.

RESULTS: Patients on ETN monotherapy $(n=2,064)$ had higher persistence $(26.8 \%$ vs. $21.1 \%$, respectively; $P<0.001$ ) on index treatment and received treatment for a longer duration (mean 375.9 days vs. 339.7 days, respectively; $P<0.001)$ than those on ADA monotherapy $(n=1,528)$. Regimen changes were more common in patients on ADA monotherapy than patients on ETN monotherapy (38.0\% vs. $33.4 \%$, respectively; $P=0.004)$. More patients on ADA monotherapy added MTX than those on ETN (17.5\% vs. $12.6 \%$, respectively; $P<0.001)$. Overall, 790 patients receiving index monotherapy had a regimen change following a gap ( $\geq 60$ days), with a similar proportion between cohorts. Among these patients, $13.8 \%$ restarted index therapy, and $7.9 \%$ switched from index therapy. Significantly more patients receiving ETN monotherapy restarted their index regimen after a gap than those receiving ADA monotherapy ( $14.9 \%$ vs. $12.2 \%$, respectively; $P=0.023$ ). The proportion of patients persistent on combination therapy was similar between the ETN and ADA combination therapy cohorts ( $21.9 \%$ vs. $22.2 \%$, respectively; $P=0.818$ ). Treatment pattern rates were similar regardless of index combination therapy. Overall, costs for ADA were consistently higher within the index regimen throughout the follow-up period irrespective of MTX.

CONCLUSIONS: ETN monotherapy as first-line treatment was associated with higher persistence, lower rate of MTX supplementation, and lower bDMARD costs than ADA monotherapy. ETN monotherapy may represent a less costly option for achieving treatment targets in bDMARD-naive patients with RA.

J Manag Care Spec Pharm. 2020;26(3):285-94

Copyright $\odot 2020$, Academy of Managed Care Pharmacy. All rights reserved.

\section{What is already known about this subject}

Traditionally, patients with rheumatoid arthritis (RA) are treated with conventional disease-modifying antirheumatic drugs (DMARDs) such as methotrexate; however, many patients do not achieve treatment targets.

Biologic DMARDs (bDMARDs), including tumor necrosis factor inhibitors (TNFi), are now indicated for the treatment of moderate to severe RA, with adalimumab and etanercept being the most commonly prescribed.

Poor adherence and low persistence to therapy in RA reduce treatment effectiveness and increase health care costs and resource utilization.

\section{What this study adds}

This study furthers our understanding of treatment patterns and health care costs among bDMARD-naive patients initiating TNFi either as monotherapy or in combination with conventional DMARDs.

Data show that patients with moderate to severe RA initiating etanercept monotherapy as first-line had higher persistence, lower rate of methotrexate supplementation, and lower bDMARD costs than those initiating adalimumab monotherapy.

First-line etanercept plus methotrexate combination therapy had similar persistence, but lower bDMARDs and total health care costs, than first-line adalimumab plus methotrexate combination therapy.

$\mathrm{R}$ heumatoid arthritis (RA) is a chronic, progressive, and disabling autoimmune disease affecting 0.6\%-1.0\% of adults in the United States. ${ }^{1}$ When unmanaged, RA may lead to joint damage, chronic pain and stiffness, loss of function, disability, and decreased life span. ${ }^{2}$

The past 15-20 years have seen significant advances in the treatment of RA, with treat-to-target strategies aimed at 


\section{Treatment Patterns and Costs in Biologic DMARD-Naive Patients with Rheumatoid Arthritis Initiating Etanercept or Adalimumab with or Without Methotrexate}

achieving low disease activity or remission as outlined in the 2015 guidelines set by the American College of Rheumatology (ACR). ${ }^{3}$ Traditionally, patients with RA are treated with conventional disease-modifying antirheumatic drugs (DMARDs) such as methotrexate (MTX). However, many patients do not achieve treatment targets. The addition of a biologic DMARD (bDMARD) or a targeted synthetic DMARD to the treatment regimen is recommended for patients with RA who have moderate or high disease activity despite monotherapy with a conventional DMARD. ${ }^{3,4}$

bDMARDs, including tumor necrosis factor inhibitors (TNFi), have transformed the course of disease for patients with moderate to severe RA. TNFis are indicated for the treatment of moderate to severe RA, ${ }^{3}$ with adalimumab (ADA) and etanercept (ETN) being the most commonly prescribed. ${ }^{5}$

Studies have demonstrated significant improvements in radiologic outcomes among patients prescribed combination therapy with TNFis plus MTX compared with MTX alone. ${ }^{6}$ However, MTX augments the efficacy of TNFis to varying extents, which may affect treatment patterns and costs. After 2 years of treatment, 59\% of patients on ADA plus MTX and 69\% of patients on ETN plus MTX combination therapy achieved $50 \%$ improvement in ACR criteria (ACR 50 response). ${ }^{7,8}$ In comparison, $37 \%$ and $48 \%$ of patients receiving ADA and ETN monotherapy, respectively, achieved an ACR 50 response., Despite clinical benefit, a decline in bDMARD use among bDMARD-naive patients from 21\% in 2006 to $18 \%$ in 2009 has been observed. Furthermore, 22\% of new bDMARD patients switched to a different bDMARD within 2 years. ${ }^{9}$

Poor adherence and low persistence to therapy in RA reduce treatment effectiveness and increase health care costs and resource utilization. ${ }^{10}$ Additionally, monthly total health care costs have been shown to be higher for patients with RA who switch therapy than for those who do not. ${ }^{11}$ Although patients receiving TNFi and MTX combination therapy have been shown to be less likely to discontinue treatment for efficacy reasons, ${ }^{12}$ data are lacking on treatment patterns and costs of TNFi as monotherapies or in combination with MTX, especially over a longer period (24 months).

Our aim was to explore real-world treatment patterns among bDMARD-naive patients with RA, examining cost differences across TNFi monotherapy and TNFi in combination with MTX. Our study focused on bDMARD-naive patients receiving their first prescription of ADA or ETN and evaluated the treatment patterns and costs as either a monotherapy or combination therapy with MTX.

\section{Methods}

\section{Study Design}

Retrospective analyses were performed using the IBM MarketScan Commercial Claims and Encounters and Medicare Supplemental Databases for the time span of January 1, 2010, through June 30, 2017. These databases include claims for approximately 137.6 million employees and their dependents and 10.2 million retirees with Medicare supplemental insurance paid for by employers. This study used deidentified database records that were fully compliant with the U.S. Health Insurance Portability and Accountability Act (HIPAA); this compliance with HIPAA regulation meant that patient consent and Institutional Review Board approval to conduct this study were not necessary.

Eligible patients were placed into the following cohorts based on index therapy: ETN monotherapy, ADA monotherapy, ETN plus MTX combination therapy, and ADA plus MTX combination therapy. Combination therapy cohorts included all patients who initiated MTX within \pm 30 days of the treatment index date.

Study periods were defined as follows: Index date refers to the date of initial ETN or ADA claim. Pre-index period refers to the 12 months preceding the index date and was used to identify demographic and clinical characteristics and to establish a bDMARD-naive population. Follow-up period refers to the 24 months after the index date and was used for assessment of outcome measures

\section{Study Population}

Inclusion Criteria. To be included in this analysis, patients were required to be aged at least 18 years on the index date and have at least 1 outpatient pharmacy claim carrying a National Drug Code number for ETN or ADA between January 1, 2011, and June 30, 2015, and at least 1 inpatient or 2 nondiagnostic outpatient claims carrying an International Classification of Diseases, Ninth Revision, Clinical Modification (ICD-9-CM) or International Classification of Diseases, Tenth Revision, Clinical Modification (ICD-10-CM) diagnosis for RA (714.x or M05-M06, respectively) during the pre-index period. Furthermore, patients were required to have continuous enrollment for the 12-month pre-index period and at least 24 months after the index date and at least 1 refill of index bDMARD after initial treatment (Appendix A, available in online article).

Exclusion Criteria. Patients were excluded from the final analyses if they had an outpatient pharmacy or medical claim for any bDMARD during the pre-index period. Patients were also excluded if they had a medical claim with a diagnosis of psoriatic arthritis, plaque psoriasis, ankylosing spondylitis, Crohn disease, ulcerative colitis, and juvenile idiopathic arthritis or diagnosis of cancer (except nonmelanoma skin cancer) during the pre-index period through the follow-up period. Adherence and dosing information cannot be assessed accurately on a medical claim; therefore, patients were also ineligible if they had an outpatient medical claim carrying a Healthcare Common Procedure Coding System code for ADA or ETN from the index date through the end of the patient's follow-up period. 


\section{Treatment Patterns and Costs in Biologic DMARD-Naive Patients with Rheumatoid Arthritis Initiating Etanercept or Adalimumab with or Without Methotrexate}

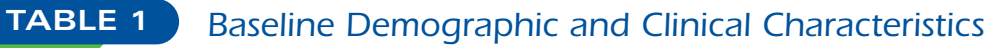

\begin{tabular}{|c|c|c|c|c|c|c|c|c|}
\hline \multirow[b]{2}{*}{ Age, years, mean (SD) } & \multicolumn{2}{|c|}{$\begin{array}{l}\text { ETN Monotherapy } \\
\qquad(\mathrm{n}=2,064)\end{array}$} & \multicolumn{2}{|c|}{$\begin{array}{l}\text { ADA Monotherapy } \\
\qquad(\mathrm{n}=1,528)\end{array}$} & \multicolumn{2}{|c|}{$\begin{array}{c}\text { ETN + MTX } \\
(n=2,134)\end{array}$} & \multicolumn{2}{|c|}{$\begin{array}{c}\mathrm{ADA}+\mathrm{MTX} \\
(\mathrm{n}=1,952)\end{array}$} \\
\hline & 50.9 & $(10.6)$ & 50.7 & $(10.4)$ & 51.8 & $(9.6)$ & 51.5 & $(9.6)$ \\
\hline Female, \% (n) & 76.3 & $(1,575)$ & 78.2 & $(1,195)$ & 77.4 & $(1,652)$ & 75.2 & $(1,467)$ \\
\hline \multicolumn{9}{|l|}{ Region, \% (n) } \\
\hline Northeast & 15.6 & $(322)$ & 14.0 & $(214)$ & 17.2 & $(367)$ & 14.3 & $(280)$ \\
\hline North central & 23.0 & $(474)$ & 22.8 & $(348)$ & 24.8 & $(529)$ & 24.9 & $(487)$ \\
\hline South & 42.9 & $(885)$ & 48.8 & $(746)$ & 39.7 & $(847)$ & 47.6 & $(929)$ \\
\hline West & 17.8 & $(368)$ & 13.8 & $(211)$ & 17.4 & $(371)$ & 12.7 & $(247)$ \\
\hline Unknown & 0.7 & (15) & 0.6 & (9) & 0.9 & $(20)$ & 0.5 & (9) \\
\hline \multicolumn{9}{|l|}{ Health plan type, \% (n) } \\
\hline Comprehensive/indemnity & 9.1 & (188) & 9.7 & $(148)$ & 7.8 & $(167)$ & 7.9 & $(155)$ \\
\hline $\mathrm{EPO} / \mathrm{PPO}$ & 58.8 & $(1,213)$ & 59.9 & (916) & 61.4 & $(1,311)$ & 61.0 & $(1,190)$ \\
\hline POS/POS with capitation & 8.0 & (166) & 9.7 & (148) & 7.6 & (163) & 9.7 & (189) \\
\hline $\mathrm{HMO}$ & 12.3 & $(253)$ & 9.8 & $(149)$ & 12.1 & $(259)$ & 9.4 & $(183)$ \\
\hline CDHP/HDHP & 10.5 & $(216)$ & 9.6 & $(146)$ & 10.0 & $(214)$ & 10.8 & $(211)$ \\
\hline Other/unknown & 1.4 & $(28)$ & 1.4 & $(21)$ & 0.9 & $(20)$ & 1.2 & $(24)$ \\
\hline \multicolumn{9}{|l|}{ Payer, \% (n) } \\
\hline Commercial & 88.3 & $(1,822)$ & 88.2 & $(1,347)$ & 89.2 & $(1,904)$ & 91.0 & $(1,777)$ \\
\hline Medicare supplemental & 11.7 & $(242)$ & 11.8 & $(181)$ & 10.8 & $(230)$ & 9.0 & $(175)$ \\
\hline Deyo-Charlson Comorbidity Index, mean (SD) & 1.4 & $(0.9)$ & 1.3 & $(0.8)$ & 1.3 & $(0.8)$ & 1.3 & $(0.8)$ \\
\hline \multicolumn{9}{|l|}{ Concomitant medications, \% (n) } \\
\hline Non-bDMARDs & 80.2 & $(1,656)$ & 80.2 & $(1,225)$ & 99.3 & $(2,120)$ & 99.1 & $(1,934)$ \\
\hline Oral corticosteroids & 71.3 & $(1,472)$ & 70.0 & $(1,069)$ & 76.8 & $(1,638)$ & 76.4 & $(1,491)$ \\
\hline Opioids & 49.3 & $(1,017)$ & 49.9 & $(762)$ & 47.3 & $(1,010)$ & 47.8 & (933) \\
\hline
\end{tabular}

$A D A=$ adalimumab; $C D H P=$ consumer-driven health plan; $b D M A R D=$ biologic disease-modifying antirheumatic drug; EPO=exclusive provider organization; $E T N=$ etanercept; $H D H P=$ high-deductible health plan; HMO = health maintenance organization; $M T X=$ methotrexate; $P O S=$ point-of-service; $P P O=$ preferred provider organization; $S D=$ standard deviation

\section{Outcomes}

Demographic and clinical characteristics were determined from claims data in the pre-index period. We assessed treatment patterns within the follow-up period according to specific modifications to index therapy: (a) switch from index therapy with prior refill gap, (b) switch from index therapy without prior refill gap, (c) restart as switch after $\geq 60$-day gap, (d) restart index therapy after $\geq 60$-day gap, (e) MTX addition or discontinuation, and (f) discontinuation of index therapy. Medication persistence was defined as an absence of treatment modifications or gaps ( $\geq 60$ days). Medication adherence was defined as when the proportion of days medication taken as prescribed covered $>80 \%$. Frequency and magnitude of index treatment dose escalation was also assessed. Dose escalation was defined as a proportion of days' supply/metric quantity that is at least $30 \%$ lower than the value of the index claim. ${ }^{13}$

Additionally, health care utilization and costs were assessed by the type of service (inpatient, outpatient, and pharmaceutical) and reported in the following categories: (a) outpatient pharmacy RA bDMARD costs, (b) other outpatient pharmacy costs (excluding RA costs), (c) outpatient medical costs, and (d) inpatient admissions costs. Costs were assessed during the follow-up period and were reported as mean costs over the follow-up period and mean per patient per month costs by treatment pattern group. Costs were based on paid amounts of adjudicated claims, including insurer and health plan payments, and patient cost sharing in the form of copayment, deductible, and coinsurance. All costs were adjusted for inflation using the medical care component of the Consumer Price Index and standardized to 2017 U.S. dollars.

\section{Statistical Analysis}

Outcomes were stratified by index therapy. Means and standard deviations were calculated for continuous variables, while counts and proportions were calculated for categorical variables. Statistically significant differences were assessed via Student's t-tests and chi-square tests for continuous and categorical variables, respectively. Statistical testing was exploratory, and comparisons were unadjusted.

\section{Results}

\section{Patient Characteristics}

Of the 231,294 patients within the IBM MarketScan Commercial or Medicare Databases with at least 1 outpatient pharmacy claim for ADA or ETN between January 1, 2011, and June 30, 2015, 7,678 (3.3\%) patients met the study inclusion criteria 


\section{Treatment Patterns and Costs in Biologic DMARD-Naive Patients with Rheumatoid Arthritis Initiating Etanercept or Adalimumab with or Without Methotrexate}

\section{TABLE 2}

Modifications to Index Therapy: Switches, Restarts, Additions, and Discontinuations

\begin{tabular}{|c|c|c|c|c|c|c|}
\hline & $\begin{array}{c}\text { ETN Monotherapy } \\
(\mathrm{n}=2,064)\end{array}$ & $\begin{array}{c}\text { ADA Monotherapy } \\
(\mathbf{n}=1,528)\end{array}$ & $\begin{array}{c}\text { ETN vs. } \\
\text { ADA } \\
P \text { Value }\end{array}$ & $\begin{array}{c}\text { ETN + MTX } \\
(n=2,134)\end{array}$ & $\begin{array}{c}\text { ADA + MTX } \\
(n=1,952)\end{array}$ & $\begin{array}{c}\text { ETN + MTX } \\
\text { vs. } \\
\text { ADA + MTX } \\
\text { P Value }\end{array}$ \\
\hline Duration of index regimen, days, mean (SD) & $375.9(261.4)$ & $339.7(252.3)$ & $<0.001$ & $357.1(252.1)$ & $353.1(252.1)$ & 0.618 \\
\hline \multicolumn{7}{|l|}{ Treatment pattern, \% (n) } \\
\hline Persistent on index regimen & $26.8 \quad(554)$ & $21.1 \quad(323)$ & $<0.001$ & $21.9 \quad(467)$ & $22.2 \quad(433)$ & 0.818 \\
\hline Regimen changes with $<60$-day gap & $33.4 \quad(690)$ & $38.0 \quad(581)$ & 0.004 & $77.8(1,660)$ & $77.4(1,510)$ & 0.741 \\
\hline Add MTX & $12.6 \quad(261)$ & $17.5 \quad(267)$ & $<0.001$ & NA & NA & NA \\
\hline Discontinue MTX & NA & NA & NA & $36.3 \quad(775)$ & $36.7 \quad(716)$ & 0.809 \\
\hline Switch therapy with no gap to & $20.8 \quad(429)$ & $20.5 \quad(314)$ & 0.863 & $41.5 \quad(885)$ & $40.7 \quad(794)$ & 0.606 \\
\hline Different bDMARD monotherapy & $18.0 \quad(371)$ & $17.5 \quad(267)$ & 0.698 & $(22)$ & 0.4 & 0.020 \\
\hline Different bDMARD + MTX & $0.8 \quad(17)$ & 0.6 & 0.412 & $(284)$ & $(240)$ & 0.333 \\
\hline MTX monotherapy & (41) & (38) & 0.312 & $(579)$ & $28.0 \quad(546)$ & 0.549 \\
\hline Regimen changes with $\geq 60$-day gap & $22.5 \quad(465)$ & $21.3 \quad(325)$ & 0.368 & 0.1 & 0.2 & 0.716 \\
\hline Add MTX & (6) & $0.5 \quad(7)$ & 0.409 & NA & NA & NA \\
\hline Discontinue MTX & NA & NA & NA & 0 & 0.1 & 0.478 \\
\hline Restart index regimen & $14.9 \quad(307)$ & $12.2 \quad(187)$ & 0.023 & 0.1 & 0.1 & 1.000 \\
\hline Switch therapy with a gap to & $7.4 \quad(152)$ & (131) & 0.184 & $<0.1$ & 0.1 & 0.609 \\
\hline Different bDMARD monotherapy & $5.0 \quad(103)$ & $5.4 \quad(83)$ & 0.555 & $<0.1$ & 0 & 1.000 \\
\hline Different bDMARD + MTX & 0 & 0 & NA & 0 & 0 & NA \\
\hline MTX monotherapy & $2.4 \quad(49)$ & $3.1 \quad(48)$ & 0.161 & 0 & 0.1 & 0.228 \\
\hline Discontinued therapy without restarting & $16.9 \quad(349)$ & $19.3 \quad(295)$ & 0.064 & 0 & $0.1 \quad(1)$ & 0.478 \\
\hline
\end{tabular}

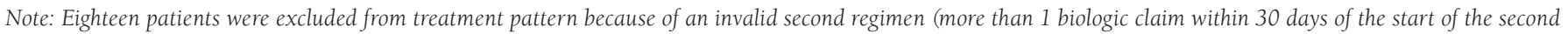
regimen). Bold results indicate statistically significant results.

$A D A=$ adalimumab; bDMARD = biologic disease-modifying antirheumatic drug; ETN=etanercept; MTX=methotrexate; NA=not applicable; SD =standard deviation.

and were evaluated (Table 1). Patients were stratified to the following cohorts according to index therapy: ETN monotherapy $(\mathrm{n}=2,064$ [26.9\%]), ADA monotherapy ( $\mathrm{n}=1,528$ [19.9\%]), ETN plus MTX ( $n=2,134$ [27.8\%]), and ADA plus MTX $(n=1,952$ [25.4\%]). Demographic and clinical characteristics were similar across cohorts. The mean patient age was 51.3 years, and $76.7 \%$ of patients were female. Most patients (73.8\%) had been prescribed oral corticosteroids, and almost half (48.5\%) had been prescribed opioids in the year before the index date. Furthermore, $80.2 \%$ of patients receiving TNFi monotherapy and $99.2 \%$ of patients receiving combination therapy had been prescribed at least 1 conventional nonbiologic DMARD (including MTX) in the year before the index date. Monotherapy cohorts did not have a prescription claim for a conventional DMARD in the 30 days before the index date.

\section{Treatment Patterns}

Monotherapy. For the 2 years after the initial ETN or ADA claim, significantly more patients in the ETN monotherapy cohort persisted on their index regimen through the end of the study than the ADA monotherapy cohort (26.8\% vs. $21.1 \%$, respectively; $P<0.001$; Table 2). Additionally, duration on the index regimen was significantly longer in the ETN monotherapy cohort compared with the ADA monotherapy cohort (mean duration: 376 days vs. 340 days, respectively;
$P<0.001$ ). Adherence (proportion of days covered $>80 \%$ ) to the index medication was similar between the ETN and ADA monotherapy cohorts ( $87.1 \%$ vs. $88.7 \%$, respectively; $P=0.129$ ). A larger proportion of patients in the ADA monotherapy cohort increased their dose by at least 30\% compared with patients in the ETN cohort $(11.2 \%$ vs. $1.8 \%$. respectively; $P<0.001)$ A larger proportion of patients receiving ADA monotherapy (19.3\%) discontinued treatment without restarting than those receiving ETN (16.9\%); however, this difference was not significant $(P=0.064)$

A larger proportion of patients receiving index ADA monotherapy had a regimen change without a prior gap ( $<60$ days) than those receiving index ETN monotherapy (38.0\% vs. $33.4 \%$, respectively; $P=0.004$ ). Among those with a regimen change without a prior gap, MTX supplementation was more frequent in the ADA monotherapy cohort than the ETN monotherapy cohort $(17.5 \%$ vs. $12.6 \%$, respectively; $P<0.001)$.

Overall, 790 patients receiving index monotherapy had a regimen change after a gap ( $\geq 60$ days), with the proportion performing similarly between cohorts (Table 2). Among these patients, 494 (13.8\%) restarted index therapy and 283 (7.9\%) switched from index therapy. Significantly more patients receiving ETN monotherapy restarted their index regimen after a gap than those receiving ADA monotherapy (14.9\% vs. $12.2 \%$, respectively; $P=0.023$ ). 


\section{A. Total Health Care Cost}

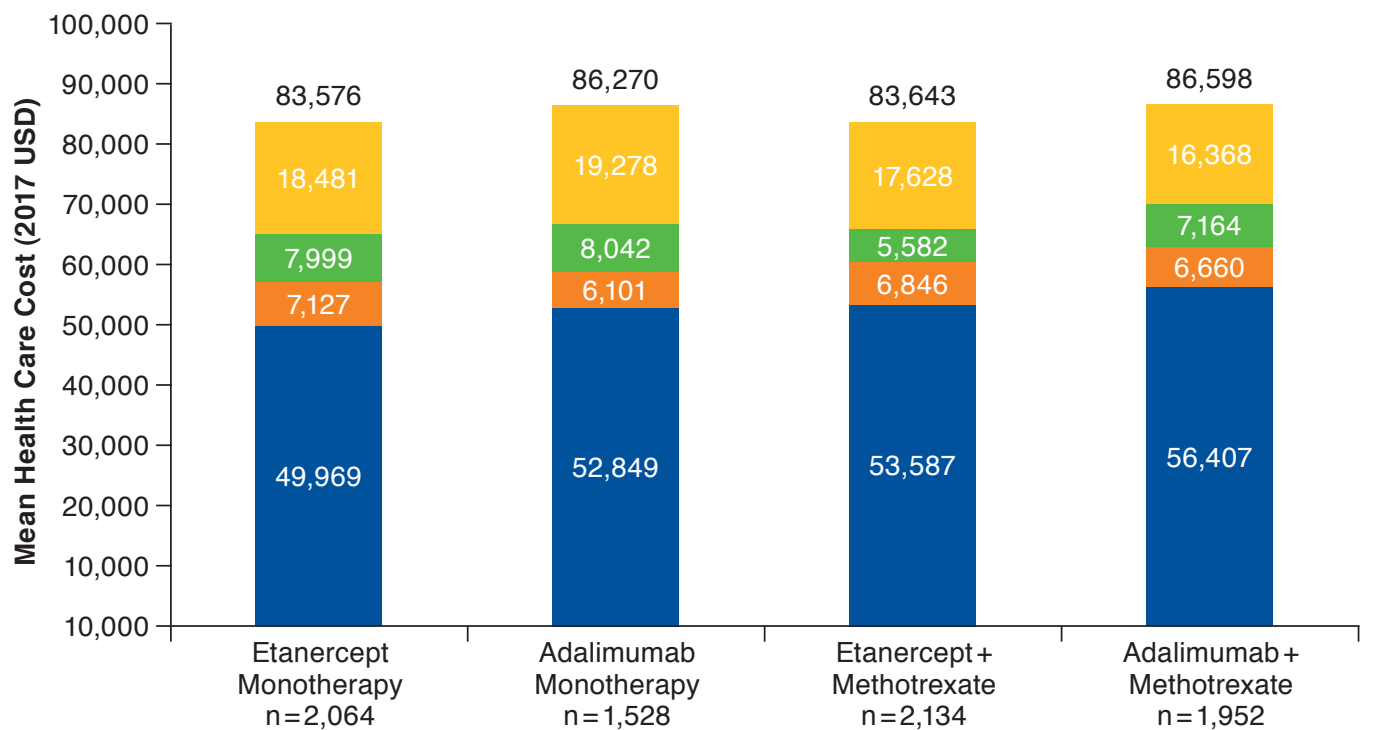

\section{B. Mean Health Care Cost PPPM for Those Who Persisted on Index Treatment}

Outpatient medical costs Inpatient admission costs Other outpatient pharmacy costs (excluding RA biologic costs)

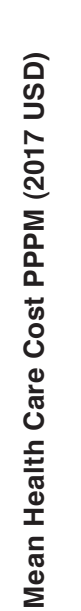

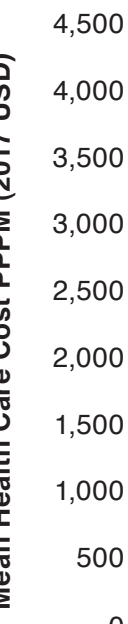
4,020 - Outpatient pharmacy RA biologic costs

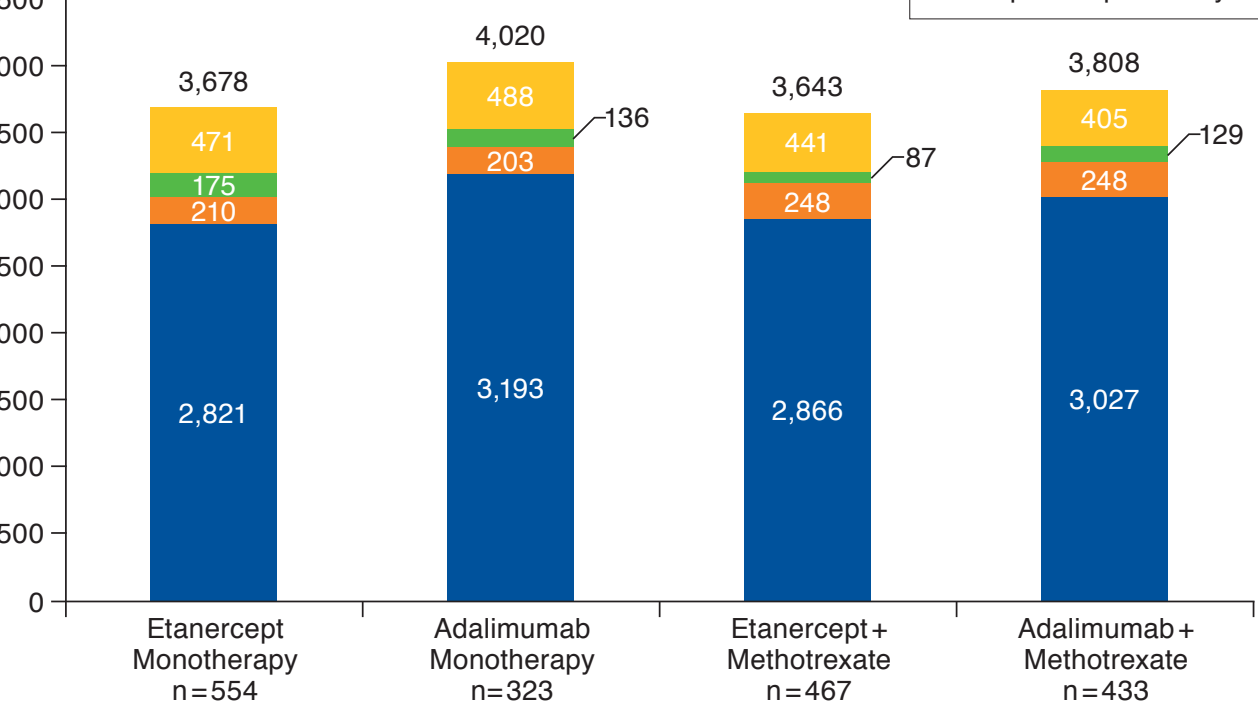

Note: Costs were based on paid amounts of adjudicated claims, including insurer and health plan payments, and patient cost sharing in the form of copayment, deductible, and coinsurance. All costs are adjusted for inflation using the medical care component of the Consumer Price Index and standardized to 2017 U.S. dollars. PPPM= per patient per month; RA=rheumatoid arthritis; USD=U.S. dollars.

Combination Therapy. The proportion of patients persistent on combination therapy was similar between the ETN and ADA combination therapy cohorts $(21.9 \%$ vs. $22.2 \%$, respectively; $P=0.818$ ). Adherence to ETN and ADA combination therapy was similar between cohorts $(64.1 \%$ vs. $63.0 \%$, respectively; $P=0.448)$. Overall, $3,170(77.6 \%)$ patients receiving index combination therapy had a regimen change without a gap, with the proportion performing similarly between cohorts 


\section{Treatment Patterns and Costs in Biologic DMARD-Naive Patients with Rheumatoid Arthritis Initiating Etanercept or Adalimumab with or Without Methotrexate}

\section{TABLE 3 Health Care and bDMARD Costs by Treatment Pattern During the 24-Month Post-Index Period}

\begin{tabular}{|c|c|c|c|c|}
\hline Treatment Pattern & ETN Monotherapy & ADA Monotherapy & $\mathrm{ETN}+\mathrm{MTX}$ & $\mathrm{ADA}+\mathrm{MTX}$ \\
\hline \multicolumn{5}{|l|}{ Mean total health care cost PPPM, USD } \\
\hline Persistent & 3,678 & 4,020 & 3,642 & 3,808 \\
\hline Add MTX & 3,376 & 3,758 & 3,356 & 3,572 \\
\hline Switch to different regimen & 3,872 & 3,825 & 3,384 & 3,416 \\
\hline Gap then restart index & 3,133 & 3,307 & 3,084 & 1,920 \\
\hline Gap then add MTXa & 3,976 & 3,914 & - & 1,924 \\
\hline Gap then switch to different regimen & 3,064 & 3,264 & 4,026 & 3,414 \\
\hline Discontinued index & 2,928 & 2,773 & - & 1,503 \\
\hline \multicolumn{5}{|c|}{ Mean outpatient pharmacy RA bDMARD cost PPPM, USD } \\
\hline Persistent & 2,821 & 3,193 & 2,866 & 3,027 \\
\hline Add MTX & 2,268 & 2,430 & 2,261 & 2,429 \\
\hline Switch to different regimen & 2,257 & 2,395 & 1,798 & 1,836 \\
\hline Gap then restart index & 1,697 & 1,854 & 2,659 & 1,647 \\
\hline Gap then add MTXa & 1,254 & 2,087 & - & 1,760 \\
\hline Gap then switch to different regimen & 1,373 & 1,414 & 497 & 1,630 \\
\hline Discontinued index & 1,022 & 1,111 & - & 700 \\
\hline
\end{tabular}

(Table 2). Among these patients, treatment pattern rates were similar regardless of index combination therapy and regimen changes after a gap were rare.

\section{Health Care Costs}

Overall, mean outpatient pharmacy RA bDMARD costs over the 24-month follow-up period were lower among the ETN cohorts ( $\$ 49,969$ monotherapy; $\$ 53,587$ plus MTX) than the ADA cohorts $(\$ 52,849$ monotherapy; $\$ 56,407$ plus MTX; $P<0.001$ for both comparisons; Figure 1A). Similarly, among patients who were persistent on their index regimen through the entire 24-month period, mean per patient per month costs and outpatient pharmacy costs for RA biologics during the 24-month follow-up period were generally lower among the ETN cohorts than the ADA cohorts (Figure 1B). Total health care costs by treatment pattern in the 24-month post-index period are shown in Table 3. Costs for ADA were consistently higher than for ETN throughout the follow-up period (Appendix B, available in online article).

\section{Discussion}

We examined the treatment patterns and health care costs from a large, nationwide database of medical and pharmacy claims of more than 7,500 bDMARD-naive patients with RA. This retrospective analysis of real-world medication use and costs of bDMARDs showed that first-line ETN monotherapy for patients with moderate to severe RA was associated with higher persistence, lower rate of MTX supplementation, and lower bDMARD costs than ADA monotherapy. Furthermore, first-line ETN plus MTX combination therapy had similar persistence but lower bDMARD and total health care costs than ADA plus MTX combination therapy. Our findings are consistent with a previous report that showed ETN had the lowest estimated bDMARD cost per effectively treated patient in a commercially insured population of patients with RA. ${ }^{14}$

Optimizing adherence and persistence with therapy is a major challenge among patients with RA, as inadequate adherence and nonpersistence with prescribed regimens can affect treatment effectiveness. ${ }^{10}$ This study found that disruptions in therapy (defined as switches, discontinuations, and restarts) were common. More than $75 \%$ of patients experienced some form of disruption during the 24 months after starting a bDMARD. The persistence rate was lower than previously reported rates..$^{15-17}$ Low persistence rates may reflect the bDMARD-naive population assessed, an inadequate response seen in patients initiating their first TNFi, or the longer followup period assessed (24 months vs. 12 months). The overall low persistence rates highlight an unmet need in existing bDMARD treatment for RA. Only a quarter of patients were persistent with their bDMARD therapies by the end of the 24-month follow-up period, whereas almost one fifth of patients ceased their monotherapy treatment. The remaining patients restarted on the same bDMARD, switched to another agent, or discontinued index therapy. Interestingly, restarting after a gap of more than 60 days was more common than switching bDMARD, which suggests that physicians and patients were inclined to 


\section{Treatment Patterns and Costs in Biologic DMARD-Naive Patients with Rheumatoid Arthritis Initiating Etanercept or Adalimumab with or Without Methotrexate}

modify index treatment using the same TNFi rather than try a different TNFi or bDMARD. This may reflect familiarity with the index treatment and a reluctance to try something different or an interruption due to an illness or surgery as opposed to an economic decision. As could be clinically expected, a larger proportion of patients in the ADA monotherapy cohort increased their dose by $\geq 30 \%$ compared with patients in the ETN cohort. This likely reflects prescribing guidance in the respective package insert in regard to the RA indication. ${ }^{18,19}$ Some patients with RA may benefit from an ADA dose increase (i.e., $40 \mathrm{mg}$ every other week to $40 \mathrm{mg}$ weekly). ${ }^{18}$ Conversely, ETN dose increases are not recommended. ${ }^{19}$

Patients with RA who switch bDMARD therapy have been shown to incur higher l-year total postswitch health care costs after the switch compared with patients who were persistent on the index bDMARD ( $\$ 44,244$ vs. $\$ 41,901 ; P=0.006$ ). Furthermore, health care costs were lowest for patients who started on ETN, particularly those who persisted on ETN $(\$ 37,638) .{ }^{20}$ Accordingly, we found that first-line ETN was associated with lower costs despite higher (ETN monotherapy) or similar (ETN combination therapy) persistence compared with ADA. Similarly, switching bDMARD therapy has been associated with increased health care costs and resource utilization among patients who do not switch to a more effective therapy. ${ }^{10,11}$

\section{Limitations}

This study was subject to limitations inherent in the analysis of administrative claims data. The analysis was limited to individuals with commercial health coverage or private Medicare supplemental coverage.

Results of this analysis may not be generalizable to bDMARD-naive patients with RA with other types of insurance or without health insurance coverage. The generalizability was also limited by the size of the analysis set, which was restricted to patients with 24-month continuous enrollment after the index date and no cancer diagnosis (15.2\% of patients with RA treated with ETN or ADA).

The potential for misclassification of RA, bDMARD use, covariates, or study outcomes was present, as patients were identified through administrative claims data as opposed to medical records. These data are subject to data coding limitations and data entry error. Misclassification of bDMARD-naive patients with RA may occur due to the 12-month pre-index period limit. It is possible that some patients may have received bDMARD therapy sometime before the 12-month pre-index period, resulting in their misclassification as bDMARD naive.

Additionally, the requirement for 24-month continuous enrollment after the index date may have led to a survival bias. Descriptive and univariate analyses were conducted for all outcomes. Analytically, we used descriptive analyses that comprise the initial, necessary phase of data exploration and insight generation, but lack the rigor of multivariate analytic approaches that may control for confounding and other relevant patient factors influencing the dependent variable of interest.

Finally, pharmacologic treatments were based on filled prescriptions with the assumption that patients take the medications; however, it cannot be confirmed if treatments were taken as prescribed.

\section{Conclusions}

ETN monotherapy as first-line treatment was associated with higher persistence, lower rate of MTX supplementation, and lower bDMARD costs than ADA monotherapy and may represent a less costly option for achieving treatment targets in bDMARD-naive patients with RA.

\section{Authors}

JOSEPH TKACZ, MS; KATHRYN HENDERSON DEYOUNG, MS; and KATHLEEN WILSON, MPH, IBM Watson Health, Cambridge, Massachusetts. MAHDI GHARAIBEH, PharmD, MS, PhD; DAVID COLLIER, MD; and HAFIZ OKO-OSI, MS, RPh, Amgen, Thousand Oaks, California.

AUTHOR CORRESPONDENCE: Joseph Tkacz, MS, IBM Watson Health, 7700 Old Georgetown Rd., Ste. 650, Bethesda, MD 20814. Tel.: 410.507.8702; E-mail: joseph.tkacz@ibm.com.

\section{DISCLOSURES}

This study was sponsored by Amgen. Tkacz, Henderson DeYoung, and Wilson are employees of IBM Watson Health, which received funding from Amgen for this study. Collier and Oko-osi are employees and shareholders of Amgen Gharaibeh was an employee of Amgen at the time of study execution and manuscript drafting.

Data pertaining to this study were presented in a poster at AMCP Nexus 2018; October 25-28, 2018; Orlando, FL.

\section{ACKNOWLEDGMENTS}

Medical writing support was provided by Jonathan Plumb, PhD, of Fishawack Communications, and funded by Amgen.

\section{REFERENCES}

1. Helmick CG, Felson DT, Lawrence RC, et al; National Arthritis Data Workgroup. Estimates of the prevalence of arthritis and other rheumatic conditions in the United States. Part I. Arthritis Rheum. 2008;58(1):15-25.

2. Pincus T, Sokka T, Wolfe F. Premature mortality in patients with rheumatoid arthritis: evolving concepts. Arthritis Rheum. 2001;44(6):1234-36.

3. Singh JA, Saag KG, Bridges SL Jr, et al. 2015 American College of Rheumatology guideline for the treatment of rheumatoid arthritis. Arthritis Rheumatol. 2016;68(1):1-26.

4. Smolen JS, Landewé R, Bijlsma J, et al. EULAR recommendations for the management of rheumatoid arthritis with synthetic and biological disease-modifying antirheumatic drugs: 2016 update. Ann Rheum Dis. 2017:76(6):960-77. 


\section{Treatment Patterns and Costs in Biologic DMARD-Naive Patients with Rheumatoid Arthritis Initiating Etanercept or Adalimumab with or Without Methotrexate}

5. Calip GS, Adimadhyam S, Xing S, Rincon JC, Lee WJ, Anguiano RH. Medication adherence and persistence over time with self-administered TNF-alpha inhibitors among young adult, middle-aged, and older patients with rheumatologic conditions. Semin Arthritis Rheum. 2017;47(2):157-64.

6. Inui K, Koike T. Combination therapy with biologic agents in rheumatic diseases: current and future prospects. Ther Adv Musculoskelet Dis. 2016; 8(5):192-202

7. Breedveld FC, Weisman MH, Kavanaugh AF, et al. The PREMIER study: a multicenter, randomized, double-blind clinical trial of combination therapy with adalimumab plus methotrexate versus methotrexate alone or adalimumab alone in patients with early, aggressive rheumatoid arthritis who had not had previous methotrexate treatment. Arthritis Rheum. 2006;54(1):26-37.

8. Klareskog L, van der Heijde D, de Jager JP, et al; TEMPO (Trial of Etanercept and Methotrexate with Radiographic Patient Outcomes) study investigators. Therapeutic effect of the combination of etanercept and methotrexate compared with each treatment alone in patients with rheumatoid arthritis: double-blind randomised controlled trial. Lancet. 2004;363(9410):675-81.

9. Crane MM, Stoykova B, Priest J, Wang N, Krzywy H, Ganguly H. Population-based analysis of treatment patterns for recently diagnosed rheumatoid arthritis patients in the United States. Paper presented at: American College of Rheumatology; October 25-30, 2013; San Diego, CA. Abstract 1050. Available at: https://acrabstracts.org/abstract/population-basedanalysis-of-treatment-patterns-for-recently-diagnosed-rheumatoid-arthritispatients-in-the-united-states/.

10. Harnett J, Wiederkehr D, Gerber R, Gruben D, Koenig A, Bourret J. Real-world evaluation of TNF-inhibitor utilization in rheumatoid arthritis. J Med Econ. 2016;19(2):91-102.

11. Meissner B, Trivedi D, You M, Rosenblatt L. Switching of biologic disease modifying anti-rheumatic drugs in patients with rheumatoid arthritis in a real world setting. J Med Econ. 2014;17(4):259-65.
12. Soliman MM, Ashcroft DM, Watson KD, Lunt M, Symmons DP, Hyrich KL; British Society for Rheumatology Biologics Register. Impact of concomitant use of DMARDs on the persistence with anti-TNF therapies in patients with rheumatoid arthritis: results from the British Society for Rheumatology Biologics Register. Ann Rheum Dis. 2011;70(4):583-89.

13. Nadkarni A, McMorrow D, Patel C, Fowler R, Smith D. Incidence of dose escalation and impact on biologic costs among patients with rheumatoid arthritis treated with three intravenous agents. J Comp Eff Res. 2017;6(8): 671-82.

14. Curtis JR, Chastek B, Becker L, et al. Cost and effectiveness of biologics for rheumatoid arthritis in a commercially insured population. J Manag Care Spec Pharm. 2015;21(4):318-29. Available at: https://www.jmcp.org/ doi/10.18553/jmcp.2015.21.4.318

15. Bonafede MMK, McMorrow D, Proudfoot C, Shinde S, Kuznik A, Chen CI. Treatment persistence and healthcare costs among patients with rheumatoid arthritis after a change in targeted therapy. Am Health Drug Benefits. 2018;11(4):192-202.

16. Bonafede M, Johnson BH, Tang DH, Shah N, Harrison DJ, Collier DH. Etanercept-methotrexate combination therapy initiators have greater adherence and persistence than triple therapy initiators with rheumatoid arthritis. Arthritis Care Res (Hoboken). 2015;67(12):1656-63.

17. Chastek B, Chen CI, Proudfoot C, Shinde S, Kuznik A, Wei A. Treatment persistence and healthcare costs among patients with rheumatoid arthritis changing biologics in the USA. Adv Ther. 2017;34(11):2422-35.

18. Humira (adalimumab) injection, solution for subcutaneous use. Abbott Laboratories. March 2011. Available at: https://www.accessdata.fda.gov/drugsatfda_docs/label/2011/125057s0276lbl.pdf. Accessed January 23, 2020.

19. Enbrel (etanercept) ) injection, for subcutaneous use. October 2019. Available at: https://www.pi.amgen.com/ /media/amgen/repositorysites/piamgen-com/enbrel/enbrel_pi.pdf. Accessed January 23, 2020.

20. Gu T, Mutebi A, Stolshek BS, Tan H. Cost of biologic treatment persistence or switching in rheumatoid arthritis. Am J Manag Care. 2018;24(8 Spec No.): SP338-45. 


\section{Treatment Patterns and Costs in Biologic DMARD-Naive Patients with Rheumatoid \\ Arthritis Initiating Etanercept or Adalimumab with or Without Methotrexate}

\section{APPENDIX A Patient Selection}

Patients in the IBM MarketScan Commercial or Medical Databases with $\geq 1$ outpatient pharmacy claim carrying a National Drug Code (NDC) number for adalimumab or etanercept from January 1, 2011, to June 30,2016 (earliest claim is the treatment index date)

Continuous enrollment with medical and pharmacy benefits in the 12 months before the treatment index date

$\geq 1$ inpatient or $\geq 2$ nondiagnostic outpatient claims carrying an ICD-9-CM or ICD-10-CM diagnosis for RA in the 12 months before or on the treatment index date

Aged at least 18 years on the treatment index date

$N=231,294$ (20)

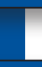

$n=130,622$

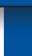

$n=50,576$

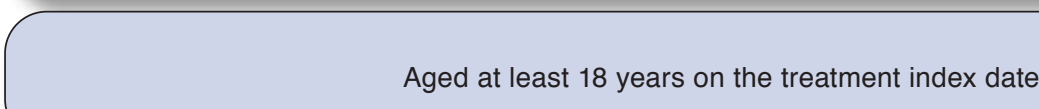

Continuous enrollment with medical and pharmacy benefits from the treatment index date through 24 months after the treatment index date

At least 1 refill for the index biologic treatment (adalimumab or etanercept) within 30 days after the run-out of the first prescription

No medical claims with a diagnosis of psoriatic arthritis, plaque psoriasis, ankylosing spondylitis,

Crohn disease, ulcerative colitis, or juvenile idiopathic arthritis during the pre-index period through the end of the patient's follow-up period

No medical claims with a diagnosis of cancer (except nonmelanoma skin cancer) during the pre-index period through the end of the patient's follow-up period

No outpatient pharmacy claim carrying an NDC number or outpatient medical claim carrying an HCPCS code for adalimumab, abatacept, anakinra, certolizumab, etanercept, golumumab, infliximab, rituximab, tocilizumab, tofacitinib in the 12 months before the treatment index date

No outpatient medical claim carrying an HCPCS code for adalimumab or etanercept from the index date through the end of the patient's follow-up period

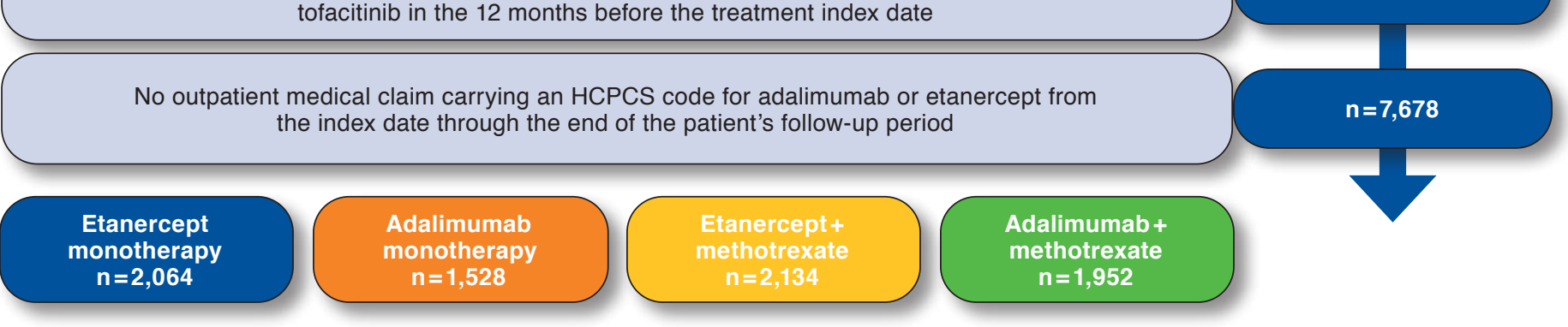

Note: Combination therapy cohorts included all patients who initiated MTX within \pm 30 days of the treatment index date.

HCPCS = Healthcare Common Procedure Coding System; ICD-9-CM=International Classification of Diseases, Ninth Revision, Clinical Modification; ICD-10-CM = International Classification of Diseases, Tenth Revision, Clinical Modification; MTX=methotrexate; RA=rheumatoid arthritis. 


\section{Treatment Patterns and Costs in Biologic DMARD-Naive Patients with Rheumatoid Arthritis Initiating Etanercept or Adalimumab with or Without Methotrexate}

\section{APPENDIX B PPPM Health Care Resource Utilization and Costs (2017 USD) over the 24-Month Post-Index}

Period, Segmented by Quarter

\begin{tabular}{|c|c|c|c|c|c|}
\hline & & $\begin{array}{c}\text { ETN Monotherapy } \\
(n=2,064)\end{array}$ & $\begin{array}{l}\text { ADA Monotherapy } \\
(\mathbf{n}=1,528)\end{array}$ & $\begin{array}{c}\text { ETN + MTX } \\
(n=2,134)\end{array}$ & $\begin{array}{c}\text { ADA + MTX } \\
(n=1,952)\end{array}$ \\
\hline \multirow{4}{*}{ Q1 } & Outpatient pharmacy RA bDMARD costs & 9,515 & 9,921 & 9,605 & 9,876 \\
\hline & Other outpatient pharmacy costs & 770 & 707 & 863 & 780 \\
\hline & Inpatient admissions costs & 502 & 536 & 358 & 221 \\
\hline & Outpatient medical costs & 1,677 & 1,687 & 1,509 & 1,393 \\
\hline \multirow{4}{*}{ Q2 } & Outpatient pharmacy RA bDMARD costs & 6,406 & 6,949 & 6,768 & 7,182 \\
\hline & Other outpatient pharmacy costs & 758 & 755 & 867 & 770 \\
\hline & Inpatient admissions costs & 608 & 882 & 675 & 1,144 \\
\hline & Outpatient medical costs & 2,057 & 2,125 & 1,960 & 1,938 \\
\hline \multirow{4}{*}{ Q3 } & Outpatient pharmacy RA bDMARD costs & 5,976 & 6,419 & 6,322 & 6,833 \\
\hline & Other outpatient pharmacy costs & 786 & 742 & 859 & 805 \\
\hline & Inpatient admissions costs & 938 & 815 & 991 & 1,144 \\
\hline & Outpatient medical costs & 2,201 & 2,410 & 2,028 & 1,942 \\
\hline \multirow{4}{*}{ Q4 } & Outpatient pharmacy RA bDMARD costs & 5,836 & 6,147 & 6,413 & 6,640 \\
\hline & Other outpatient pharmacy costs & 895 & 730 & 840 & 862 \\
\hline & Inpatient admissions costs & 1,643 & 916 & 821 & 688 \\
\hline & Outpatient medical costs & 2,423 & 2,705 & 2,119 & 2,097 \\
\hline \multirow{4}{*}{ Q5 } & Outpatient pharmacy RA bDMARD costs & 5,485 & 5,862 & 6,026 & 6,368 \\
\hline & Other outpatient pharmacy costs & 906 & 725 & 815 & 834 \\
\hline & Inpatient admissions costs & 840 & 982 & 631 & 807 \\
\hline & Outpatient medical costs & 2,151 & 2,452 & 2,406 & 2,229 \\
\hline \multirow{4}{*}{ Q6 } & Outpatient pharmacy RA bDMARD costs & 5,565 & 5,984 & 6,021 & 6,436 \\
\hline & Other outpatient pharmacy costs & 930 & 858 & 854 & 844 \\
\hline & Inpatient admissions costs & 1,264 & 1,522 & 889 & 1,187 \\
\hline & Outpatient medical costs & 2,478 & 2,579 & 2,394 & 2,194 \\
\hline \multirow{4}{*}{ Q7 } & Outpatient pharmacy RA bDMARD costs & 5,545 & 5,686 & 6,100 & 6,501 \\
\hline & Other outpatient pharmacy costs & 932 & 779 & 862 & 836 \\
\hline & Inpatient admissions costs & 1,145 & 1,242 & 579 & 1,091 \\
\hline & Outpatient medical costs & 2,655 & 2,597 & 2,668 & 2,323 \\
\hline \multirow{4}{*}{ Q8 } & Outpatient pharmacy RA bDMARD costs & 5,640 & 5,880 & 6,331 & 6,573 \\
\hline & Other outpatient pharmacy costs & 1,150 & 807 & 886 & 927 \\
\hline & Inpatient admissions costs & 1,059 & 1,148 & 638 & 883 \\
\hline & Outpatient medical costs & 2,839 & 2,723 & 2,543 & 2,252 \\
\hline
\end{tabular}

$A D A=$ adalimumab; $b D M A R D=$ biologic disease-modifying antirheumatic drug; ETN=etanercept; $M T X=$ methotrexate; PPPM = per patient per month; $R A=$ rheumatoid arthritis; USD $=$ U.S. dollars. 
